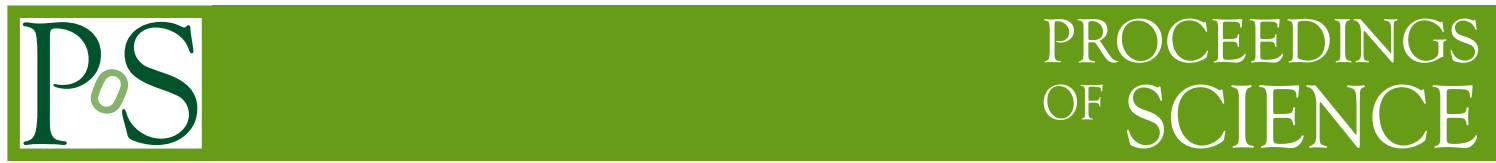

\title{
Electroweak boson production at LHCb
}

\section{S. Tourneur*, on behalf of the LHCb Collaboration}

Ecole Polytechnique Fédérale de Lausanne, Switzerland

E-mail: stephane.tourneur@cern.ch

We report measurements of electroweak boson production in the forward region, using protonproton collison data collected at the LHCb experiment at a centre of mass energy of $\sqrt{s}=7 \mathrm{TeV}$ with an integrated luminosity of up to $1.0 \mathrm{fb}^{-1}$. W and $\mathrm{Z}$ bosons are reconstructed in leptonic decay channels, and their cross-sections determined using data-driven techniques. All results are compared to NLO and NNLO predictions.

XXI International Workshop on Deep-Inelastic Scattering and Related Subject-DIS2013, 22-26 April 2013

Marseilles, France

\footnotetext{
${ }^{*}$ Speaker.
} 


\section{Introduction}

Measurements of differential cross-sections for $\mathrm{W}$ and $\mathrm{Z}$ boson production at the Large Hadron Collider (LHC) provide important tests of the proton's Parton Distribution Functions (PDFs) and partonic cross-section calculations at next-to-leading-order (NLO) and next-to-next-to-leadingorder (NNLO) from QCD. While the latter are known at precision levels of few percent, the knowledge of the PDFs is the biggest source of uncertainty to the electroweak boson cross-sections at LHC. Their uncertainty for $\mathrm{W}$ and $\mathrm{Z}$ production is about $2 \%$ in the central region but increases towards the forward region[1]. Since the LHCb detector[2] is the only detector at the LHC to be fully instrumented at high pseudorapidities $(\eta)$ between 2 and 5 where the uncertainties are the largest, it constitutes a very interesting place to perform such measurements. Indeed, for a $\mathrm{W}$ or $\mathrm{Z}$ boson to be produced with enough forward boost to decay inside the LHCb detector, a high Bjorken-X quark from one proton needs to collide against a low Bjorken-x quark from the other proton, therefore probing the proton's PDF at both low and high $\mathrm{x}$. Fig. 1 shows which regions of the $\left(\mathrm{x}, \mathrm{Q}^{2}\right)$ space the $\mathrm{LHCb}$ experiment is sensitive to in comparison with other past and present experiments. $\mathrm{Q}$ is the momentum scale of the process. It is worth to stress that $\mathrm{LHCb}$ is the first and only experiment that enables to explore low $\mathrm{x}$ regions $\left(10^{-5}<\mathrm{x}<10^{-3}\right)$ at $\mathrm{Q}^{2}>10 \mathrm{GeV}^{2}$.

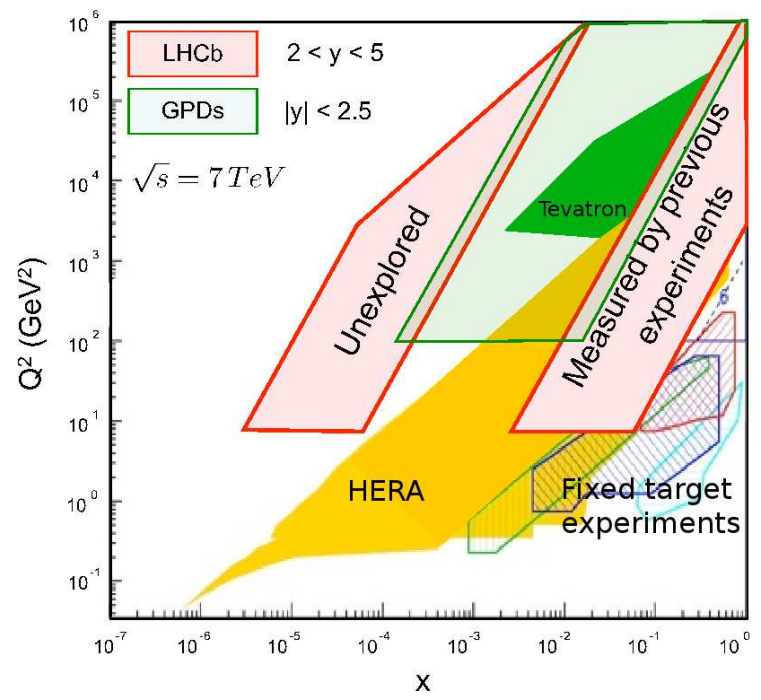

Figure 1: The regions of the $\left(\mathrm{x}, \mathrm{Q}^{2}\right)$ space probed at LHCb compared with ATLAS, CMS (GPDs in the figure) and several past experiments.

The following cross-section measurements at $\mathrm{LHCb}$ include the analysis of the $\mathrm{Z}$ and $\mathrm{W}$ boson production in $p p$ collisions at $\sqrt{s}=7 \mathrm{TeV}$ in the $Z \rightarrow \mu^{+} \mu^{-}, Z \rightarrow e^{+} e^{-}, Z \rightarrow \tau^{+} \tau^{-}$and $\mathrm{W} \rightarrow \mu \nu$ decays.

\section{Event selection and background subtraction}

All Z measurements make use of a data sample corresponding to an integrated luminosity of $1 \mathrm{fb}^{-1}$ collected with the LHCb detector, whereas the $\mathrm{W}$ measurement is based on $37 \mathrm{pb}^{-1}$. 


\section{$2.1 Z \rightarrow \mu^{+} \mu^{-}$events}

In the dimuon channel, $\mathrm{Z}$ events are triggered with the online requirement of a single muon with a transverse momentum $\left(p_{T}\right)$ larger than $10 \mathrm{GeV} / \mathrm{c}$. Muons are identified using a muon system composed of alternating layers of iron and multiwire proportional chambers.

The offline selection then requires two oppositely-charged muon candidates, both with $\eta$ between 2 and 4.5 and $p_{T}$ above $20 \mathrm{GeV} / \mathrm{c}$. In order to select events compatible with $\mathrm{Z}$ boson production, only dimuon pairs whose invariant mass is between 60 and $120 \mathrm{GeV} / \mathrm{c}^{2}$ are kept. The final event selection retains $52626 \mathrm{Z} \rightarrow \mu \mu$ candidate events. Their invariant mass distribution is shown in Fig. 2.

The background events due to heavy flavour hadrons that decay semileptonically or due to mis-identification of hadrons (mesons decaying to muons or punching through the calorimeters up to the outer muon chambers) are measured using data-driven methods. Backgrounds due to a partial reconstruction of top quark pairs, diboson or $Z \rightarrow \tau \tau$ events, are estimated using a PYTHIA 6.4[3] simulation.

A total of $153 \pm 14$ background events are found to contaminate the selected sample, which corresponds to a purity ${ }^{1}$ of $0.9971 \pm 0.0003$ [4].

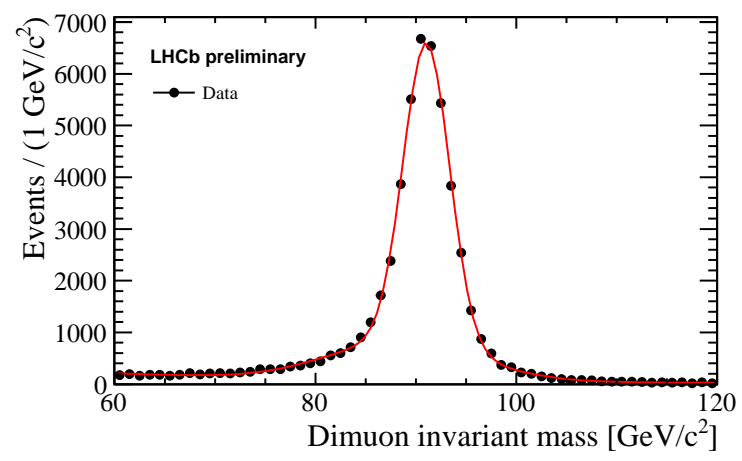

Figure 2: Distribution of the invariant mass of the dimuon pairs in the selected $Z \rightarrow \mu^{+} \mu^{-}$events.

\section{2 $Z \rightarrow e^{+} e^{-}$events}

In the dielectron channel, $\mathrm{Z}$ events are triggered with the online requirement of a single electron with $p_{T}$ larger than 10 or $15 \mathrm{GeV} / \mathrm{c}$ depending on the data taking period. Electrons are identified by combining information from the tracker system and from the shower profile in the electromagnetic and hadronic calorimeters.

The offline analysis then requires two oppositely-charged electron candidates, both with $\eta$ between 2 and 4.5 and $p_{T}$ above $20 \mathrm{GeV} / \mathrm{c}$. In order to select events compatible with $\mathrm{Z}$ boson production, only dielectron pairs whose measured invariant mass is above $40 \mathrm{GeV} / \mathrm{c}^{2}$ are kept.

A data sample of same-sign $\mathrm{e}^{ \pm} \mathrm{e}^{ \pm}$pairs provides a data-based estimate of background which is found to account for $4.5 \%$ of the selected sample[5].

\footnotetext{
${ }^{1}$ The purity is defined as the ratio of the numbers of signal to candidate events.
} 


\section{$2.3 Z \rightarrow \tau^{+} \tau^{-}$events}

In the $\tau \tau$ channel, $\mathrm{Z}$ events are triggered with the online requirement of a single muon with $p_{T}$ larger than $10 \mathrm{GeV} / \mathrm{c}$ or a single electron with $p_{T}$ larger than $15 \mathrm{GeV} / \mathrm{c}$.

Selected $Z \rightarrow \tau^{+} \tau^{-}$candidate events have two oppositely-charged tracks, consistent with an electron, muon, or 1-prong hadron $\tau$ decay. The two tracks are required to have a large impact parameter (IP) with respect to the primary vertex of the event in order to decrease the prompt background. At least one of the two tracks has to correspond to an identified electron or muon with $p_{T}$ larger than $20 \mathrm{GeV} / \mathrm{c}$ and $\eta$ between 2 and 4.5. The second track must have $p_{T}$ larger than 5 $\mathrm{GeV} / \mathrm{c}$. Its $\eta$ must be between 2 and 4.5 in case of an electron or muon and between 2.25 and 3.75 in case of a hadron. $\tau$ pairs with two electronic decays are not considered.

The main backgrounds involve generic QCD and W/Z+jets events that have jets faking the $\tau$ signatures. These are estimated using same-sign $\tau^{ \pm} \tau^{ \pm}$data.

The purities of the selected samples are found to lie between 65 and $70 \%$ for all channels[6].

\section{$2.4 \mathrm{~W} \rightarrow \mu v$ events}

As much as $16 \%$ of $W \rightarrow \mu v$ events produced in $p p$ collisions at $7 \mathrm{TeV}$ at the LHCb interaction point have the muon emitted inside the LHCb acceptance. Although no reconstruction of the missing energy is feasible with LHCb, a selection of an abundant and rather pure $\mathrm{W} \rightarrow \mu \nu$ events by the $\mathrm{LHCb}$ experiment is still possible.

$\mathrm{W}$ events are triggered with the online requirement of a single muon with a transverse momentum larger than $10 \mathrm{GeV} / \mathrm{c}$.

The offline selection of $W \rightarrow \mu v$ events proceeds with the identification of a muon defined as in the $Z \rightarrow \mu \mu$ analysis. It is required to have $p_{T}$ between 20 and $70 \mathrm{GeV} / \mathrm{c}$ and $\eta$ between 2.0 and 4.5. Several other cuts are applied to increase the purity. The muon is required to be isolated and with a small IP with respect to the primary vertex of the event to decrease the heavy-flavour background. A reduced fraction $(<4 \%)$ of the muon energy is allowed to be deposited in the calorimeters to decrease the background from pions and kaons. Finally events presenting a second muon with $p_{T}>2 \mathrm{GeV} / \mathrm{c}$ are discarded to decrease the $Z \rightarrow \mu \mu$ background. The event selection eventually retains $14660 \mathrm{~W}^{+}$and $11618 \mathrm{~W}^{-}$candidate events[7].

The two most important backgrounds are $Z \rightarrow \mu \mu$ events and generic QCD events where a kaon or pion decays leptonically before reaching the muon chambers (" $K / \pi \rightarrow \mu v$ "). The purity of the selected sample is obtained from a template fit to the muon $p_{T}$ distribution as shown in Fig. 3. The only parameters allowed to vary in the fit are the normalizations of the $\mathrm{W}$ signal and $K / \pi \rightarrow \mu \nu$ background, whereas the $p_{T}$ shapes of all contributions are fixed[7]. A purity close to $80 \%$ is found.

\section{Efficiencies}

The efficiencies of the track reconstruction, electron identification, muon triggering and identification, are estimated using tag-and-probe methods in $Z \rightarrow l^{+} l^{-}$data samples. This technique profits from the very high purity of $Z \rightarrow l^{+} l^{-}$event samples to isolate a pure and minimally biased sample of true leptons that can be used to compute the lepton-related efficiencies in data itself. All efficiencies are determined as a function of the lepton $\eta[4][5][6][7]$. 


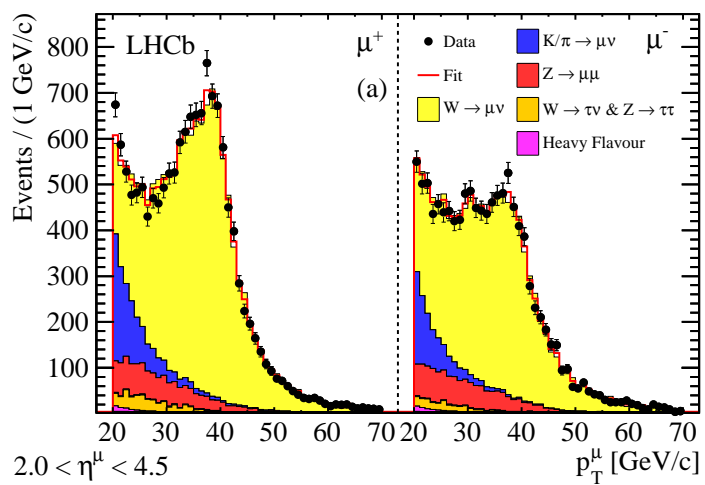

Figure 3: Distribution of the muon $p_{T}$ for the selected $W^{+} \rightarrow \mu^{+} v$ candidate events (left) and the selected $W^{-} \rightarrow \mu^{-} v$ candidate events (right)[7]. The $\mathrm{W}$ signal and different background contributions as well as the result of the fit are shown in the plots.

\section{Results}

The knowledge of the efficiencies, luminosity[8] and purity enables the total cross-sections to be computed from the observed numbers of events. The cross-section is also determined as a function of $\eta$ for the $\mathrm{W}$ sample and as a function of the $\mathrm{Z}$ rapidity (y) and $\phi^{* 2}$ of the $\mathrm{Z}$ boson for $Z \rightarrow \mu \mu$ and $Z \rightarrow e e$, as well as $Z p_{T}$ for the $Z \rightarrow \mu \mu$ channel. The cross-sections are corrected to Born level in quantum electrodynamics to be consistent with compared QCD predictions which do not include the effects of final state radiation. They are further corrected with PYTHIA to take into account the bin-to-bin migrations.

All measured cross-sections are calculated relative to the following fiducial and kinematical volume chosen identical across all electroweak analyses: $p_{T}>20 \mathrm{GeV} / \mathrm{c}$ and $2<\eta<4.5$ for the $e$, $\mu$ or $\tau$, and in case of measurements of the $Z$ boson, a dilepton mass between 60 and $120 \mathrm{GeV} / \mathrm{c}^{2}$.

The measured differential cross-sections for the $Z \rightarrow \mu \mu$ production at $7 \mathrm{TeV}$ as a function of the $\mathrm{Z}$ rapidity are found to be in agreement with NNLO theoretical predictions using several sets[10][11][12] for the PDFs of the proton (Fig. 4). After extrapolation to the ATLAS fiducial volume with FEWZ[13] at NLO, the Z cross-section measured at LHCb is also compared and found in agreement with ATLAS results[14] in the overlapping regions of rapidity in Fig. 4[15]. This figure also shows the good agreement obtained between the $\mathrm{Z}$ measurements at $\mathrm{LHCb}$ in the dielectron and dimuon channels.

The measured differential cross-sections for the $Z \rightarrow \mu \mu$ production at $7 \mathrm{TeV}$ as a function of the $p_{T}$ of the $\mathrm{Z}$ boson are found to be in better agreement with predictions including parton showering (Powheg[16]+PYTHIA) or QCD resummation (ResBos[17]) than with fixed-order calculations at NNLO (FEWZ), as can be seen in the plot of the differential cross-section of Fig. 5.

The integrated cross-sections for $Z \rightarrow \mu \mu, Z \rightarrow e e$ and $Z \rightarrow \tau \tau$ are shown together in Fig. 5 . They are all consistent with each other and in agreement with the NNLO prediction from FEWZ using the MSTW08[10] PDF set.

\footnotetext{
${ }^{2} \phi^{*}$ is a variable close to the ratio $p_{T} / M$ of a reconstructed pair of particles and that presents the advantage of being less dependent on the momentum resolution.[9][5]
} 

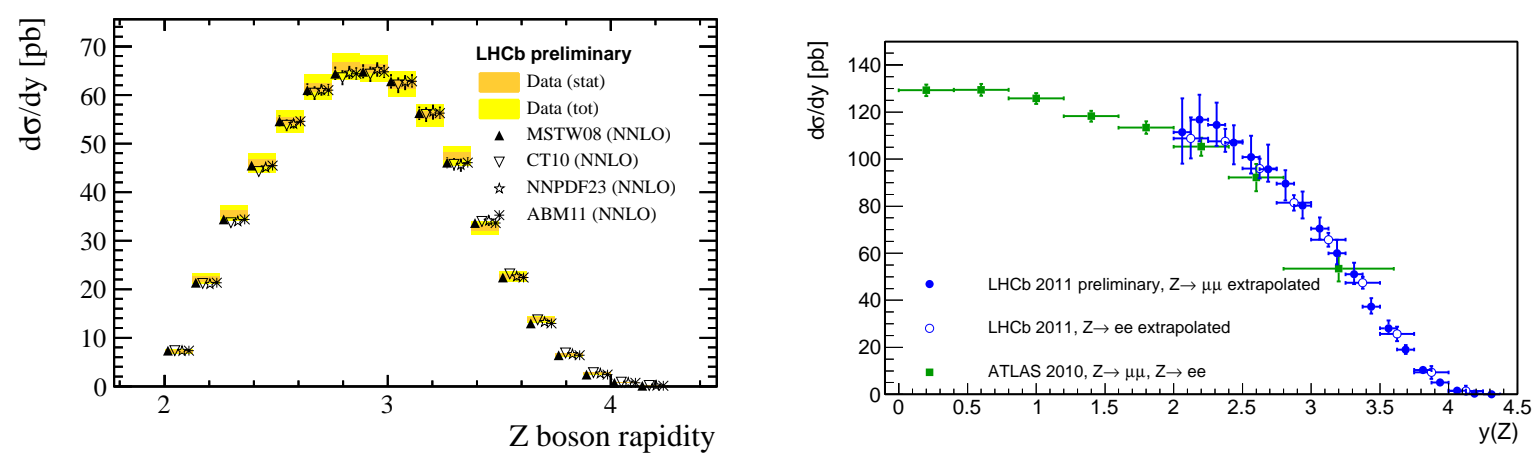

Figure 4: Differential $Z \rightarrow \mu \mu$ cross-sections as a function of the rapidity of the $Z$ boson. Left: in the LHCb fiducial volume region[4]. Predictions at NNLO using several sets[10][11][12] for the PDF are superimposed. Right: Comparison of the ATLAS cross-section measurement[14] with the LHCb cross-section measurements in the dielectron and dimuon channels extrapolated to the ATLAS fiducial volume[15].
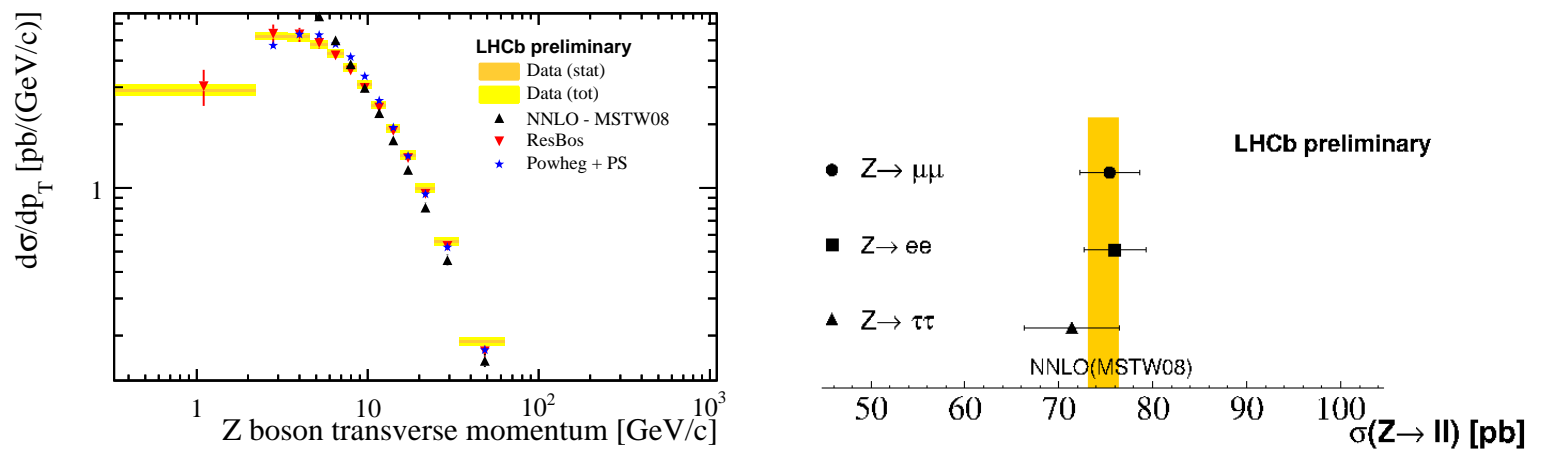

Figure 5: Left: Differential $Z \rightarrow \mu \mu$ cross-sections as a function of the $p_{T}$ of the $\mathrm{Z}$ boson compared with perturbative predictions at NNLO[13], predictions from the QCD resummation program ResBos[17] and PowHeg[16]+PYTHIA[3]. Right: Summary of the LHCb measurements of $\sigma(Z \rightarrow l l)$ for $1=e, \mu, \tau$ at $\sqrt{s}$ $=7 \mathrm{TeV}[4]$. The prediction from MSTW08[10] with its uncertainty is superimposed.

Some results for the measurements of the differential $\mathrm{W}$ cross-section and lepton charge asymmetry (defined as $\left(\sigma\left(W^{+} \rightarrow l^{+} v\right)-\sigma\left(W^{-} \rightarrow l^{-} v\right)\right) /\left(\sigma\left(W^{+} \rightarrow l^{+} v\right)+\sigma\left(W^{-} \rightarrow l^{-} v\right)\right)$ obtained with $37 p b^{-1}$ of data are shown in Fig. 6. In order to provide a comparison with ATLAS and CMS measurements, the LHCb results are corrected to match the fiducial cuts used in the other experiments and a very good agreement is observed in the overlapping range of pseudorapidities. All measured total $W^{+,-}$and $Z$ cross-sections and ratios have been found in agreement with NLO and NNLO predictions using different PDF sets[7].

\section{Conclusion}

The inclusive and differential cross-sections have been presented for the forward production of $\mathrm{Z}$ and $\mathrm{W}$ bosons in $p p$ collisions at a centre of mass energy of $\sqrt{s}=7 \mathrm{TeV}$. The measured $\mathrm{Z}$, $\mathrm{W}^{+}$and $\mathrm{W}^{-}$total cross-sections, as well as ratios and lepton charge asymmetry are all compatible 

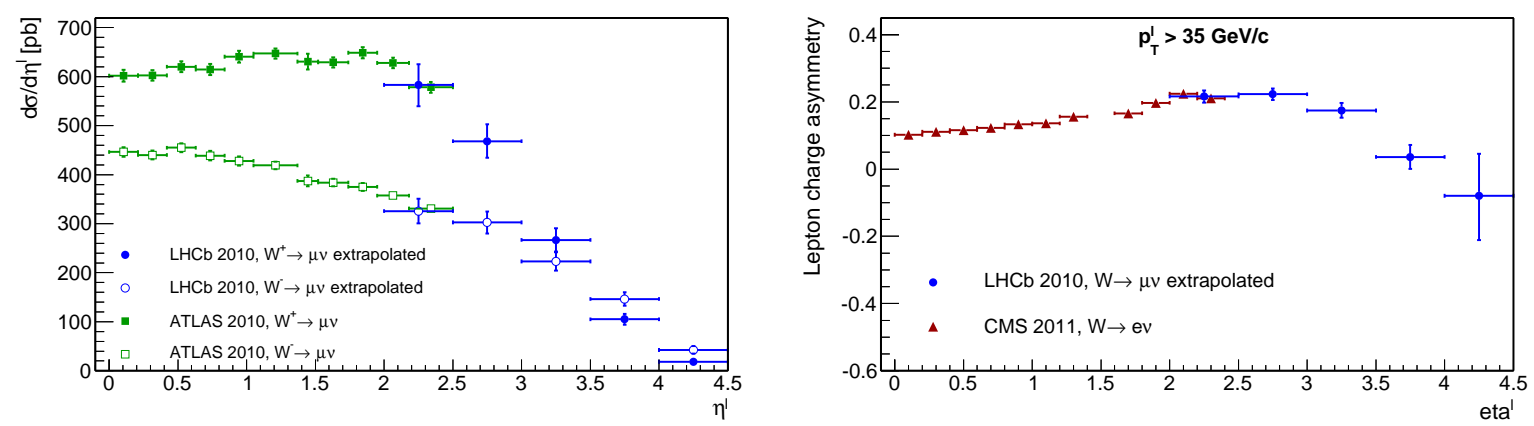

Figure 6: Left: Differential $W^{+,-} \rightarrow \mu^{+,-} v$ cross-sections as a function of the muon $\eta$ extrapolated and compared with ATLAS[14]. Right: Lepton charge asymmetries as a function of the lepton $\eta$ measured at LHCb and CMS[18] at $7 \mathrm{TeV}$. The LHCb results are corrected to match the fiducial cuts of ATLAS and CMS, respectively[15].

with NLO and NNLO predictions using different PDF sets. The electroweak boson cross-sections have also been measured as a function of the $\mathrm{Z}$ rapidity or muon pseudorapidity and no significant disagreement has been found with respect to fixed-order predictions using different parametrisations for the PDF. In the case of the differential $\mathrm{Z}$ cross-section as a function of the $\mathrm{Z}$ transverse momentum, predictions using resummation or parton shower programs reproduce the LHCb data better than perturbative calculations at NNLO.

The precisions of all measurements relative to the electroweak boson production at $\mathrm{LHCb}$ are expected to improve significantly with further data as their present systematic uncertainties are mostly statistical in nature.

\section{References}

[1] R.S. Thorne et al., Parton Distributions and QCD at LHCb, [arXiv: 0808.1847].

[2] LHCb collaboration, The LHCb Detector at the LHC, JINST 3 (2008) S08005.

[3] T. Sjöstrand et al., PYTHIA 6.4 physics and manual, JHEP 05 (2006) 026 [hep-ex / 0603175$].$

[4] LHCb collaboration, Measurement of the cross-section for $Z \rightarrow \mu^{+} \mu^{-}$production with $1 \mathrm{fb}^{-1}$ of pp collisions at $\sqrt{s}=7 \mathrm{TeV}$, LHCb-CONF-2013-007.

[5] LHCb collaboration, Measurement of the cross-section for $\mathrm{Z} \rightarrow e^{+} e^{-}$production in pp collisions at $\sqrt{s}=7 \mathrm{TeV}$, JHEP 02 (2013) 106 [arXiv:1212 . 4620].

[6] LHCb collaboration, A study of the Z production cross-section in pp collisions at $\sqrt{s}=7 \mathrm{TeV}$ using tau final states, JHEP 01 (2013) 111 [arXiv:1210 . 6289].

[7] LHCb collaboration, Inclusive $W$ and $Z$ production in the forward region at $\sqrt{s}=7 \mathrm{TeV}$, JHEP 06 (2012) 058 [arXiv:1204.1620].

[8] LHCb collaboration, Absolute luminosity measurements with the LHCb detector at the LHC, JINST 7 (2012) P01010 [arXiv: 1110.2866$].$

[9] A. Banfi et al., Optimisation of variables for studying dilepton transverse momentum distributions at hadron colliders, Eur. Phys. J. C71 (2011) 1600 [arXiv:1009.1580]. 
[10] A. D. Martinet al., Parton distributions for the LHC, Eur. Phys. J. C63 (2009) 189 [arXiv:0901.0002].

[11] J. G. et. al., The CT10 NNLO Global Analysis of QCD [arXiv: 1302 . 624 6].

[12] R. D. Ball et al., A first unbiased global NLO determination of parton distributions and their uncertainties, Nucl. Phys. B838 (2010) 136 [arXiv: 1002 . 4407 ].

[13] R. Gavin et al., FEWZ 2.0: A code for hadronic Z production at next-to-next-to-leading order, Comput. Phys. Commun. 182 (2011) 2388 [arXiv: 1011.3540 ].

[14] ATLAS collaboration, Measurement of the $W \rightarrow l v$ and $Z / \gamma^{*} \rightarrow l l$ production cross sections in proton-proton collisions at $\sqrt{s}=7$ TeV with the ATLAS detector, JHEP 12 (2010) 060 [arXiv: 1010 . 2130]; JHEP 06 (2012) 058 [arXiv: 1204 . 1620].

[15] LHCb collaboration, Graphical comparison of the LHCb measurements of $W$ and Z boson production with ATLAS and CMS, LHCb-CONF-2013-005.

[16] P.Nason, A New Method for Combining NLO QCD with Shower Monte Carlo Algorithms, JHEP 0411 (2004)040 [hep-ph/ 0409146 ]; S.Frixione et al., Matching NLO QCD computations with Parton Shower simulations: the POWHEG method, JHEP 0711 (2007) 070 [arXiv: 0709 . 20 92]; S. Alioli et al., A general framework for implementing NLO calculations in shower Monte Carlo programs: the POWHEG BOX, JHEP 1006 (2010) 043 [arXiv : 1002 . 2581].

[17] G. A. Ladinsky and C.-P. Yuan, The Nonperturbative Regime in QCD Resummation for Gauge Boson Production at Hadron Colliders, Phys. Rev. D50 (1994) 4239 [hep-ph/9311341]; C. Balazs and C.-P. Yuan, Soft gluon effects on lepton pairs at hadron colliders, Phys. Rev. D56 (1997) 5558 [hep-ph/9704258]; F. Landry et al., Tevatron Run-1 Z Boson Data and Collins-Soper-Sterman Resummation Formalism, Phys. Rev. D67 (2003) 073016 [hep-ph / 0212159 ].

[18] CMS collaboration, Measurement of the electron charge asymmetry in inclusive W production in pp collisions at sqrt(s) = 7 TeV, Phys. Rev. Lett. 169109 (2012) 111806 [arXiv:1206.2598]. 